\title{
Efecto de la aplicación de fibra de coco (Cocos nucifera L.) en el almacenamiento y eficiencia del uso del agua en un Alfisol, sembrado con ballica (Lolium multiflorum L.) y en la toxicidad en lechuga (Lactuca sativa L.).
}

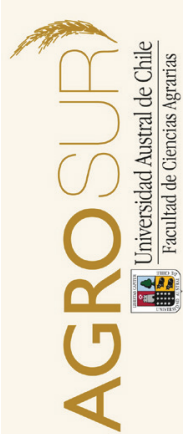

\author{
Effect of applying coconut fiber (Cocos nucifera L.) on storage and water use \\ efficiency in an Alfisol seeded with ryegrass (Lolium multiflorum L.) and its \\ toxicity in lettuce (Lactuca sativa L.).
}

\author{
Sandoval, M. ${ }^{a^{*}}$, Zapata, M. ${ }^{a}$, Celis, J. ${ }^{b}$, Quezada, C. ${ }^{a}$, Capulín, J. ${ }^{c}$, Solís, A. ${ }^{a}$ \\ ${ }^{a}$ Universidad de Concepción, Facultad de Agronomía, Departamento de Suelos y Recursos Naturales. \\ Avda. Vicente Méndez 595, Chillán. \\ ${ }^{b}$ Departamento de Ciencias Pecuarias, Universidad de Concepción, Av. Vicente Méndez 595, Chillán, Chile. \\ ${ }^{c}$ Instituto de Ciencias Agropecuarias, Universidad Autónoma del Estado de Hidalgo, Km 1 s/n, Tulancingo, México.
}

ART I C LE INFO

\section{Keywords:}

Coconut fiber

Plant available water

Water use efficiency

Alfisol

Original Research Article,

Soil Science

${ }^{*}$ Corresponding author:

Marcos Sandoval

E-mail address:

masandov@udec.cl
A B S T R A C T

The search for techniques that allow a more efficient use of water is a global task, since this resource is increasingly scarce and its quality is constantly deteriorating. The objective of this study was to determine the effect of applying coconut fiber $\mathrm{CF}$ on physical-hydric parameters of the soil and its water use efficiency (WUE). The effects of coconut fiber (CF) were assessed on water consumption, water use efficiency and aerial and root biomass of Italian ryegrass (Lolium multiflorum L.). In addition, it was also evaluated whether CF causes toxic effects on lettuce seeds (Lactuca sativa $\mathrm{L}$.). Treatments were established according to the proportion of CF and soil in $\mathrm{g} \mathrm{g}^{-1}$ soil CF: T1 = 1:80, T2 $=1: 40$ and $\mathrm{T} 3=1: 20$, and a control treatment $(\mathrm{T} 0=$ no substrate $)$. Additionally, 3 charges of water $(100,75,50 \%$ of field capacity) were established. Results showed that the best treatment for water consumption was T3-50 (377.8 ml), for WUE the best treatment was T3-100 (8.52 $\left.\mathrm{kg} \mathrm{DM} \mathrm{m}^{-3}\right)$, for aerial biomass it was T3-100 (4.59 g MS) and in the case of root biomass the best treatment was T3100 (1.92 g MS). It was concluded that the additionof coconut fiber substrate to the mix improves the physical and hydrological characteristics of degraded soil. There were no toxic effects on lettuce among the applied rates of CF. The germination index (IG) ranged from 124.82 to $156.03 \%$ and the best treatments were $\mathrm{T} 3$ and $\mathrm{T} 2$.

\section{RESUMEN}

La búsqueda de alternativas que permitan un uso más eficiente del agua es una tarea mundial, puesto que este recurso es cada vez más escaso y su calidad se deteriora. De ahí que la presente investigación tuvo como objetivo determinar el efecto de la aplicación de fibra de coco (FC) sobre parámetros físico-hídricos del suelo y su eficiencia en el uso del agua (EUA). Se probaron los efectos de la FC sobre el gasto de agua, sobre la EUA, biomasa aérea y radical en ballica italiana (Lolium multiflorum L.). Además se evaluó si la FC presenta efectos tóxicos sobre las semillas de lechuga (Lactuca sativa L.). Los tratamientos fueron establecidos de acuerdo a la proporción de FC y suelo, en gramos FC g-1 suelo: T1 = 1:80, T2 = 1:40 y T3 = 1:20, y un testigo ( $\mathrm{T} 0=$ Sin sustrato). Adicionalmente, se establecieron 3 cargas de agua $(100,75,50 \%$ de capacidad de campo). Los resultados muestran que el menor consumo de agua se dio en el tratamiento T3-50 (377,8 ml), para la EUA el mejor tratamiento fue T3100 (8,52 $\mathrm{kg} \mathrm{MS} \mathrm{m}^{-3}$ ), para biomasa aérea, el mejor fue el tratamiento T3-100 (4,59 g MS) y en el caso de la biomasa radical su mejor tratamiento fue el T3-100 (1,92 g MS). Por lo tanto, al adicionar el sustrato de fibra de coco en la mezcla, este permite un mejoramiento en las características físico-hídricas del suelo degradado. Las pruebas con lechuga indicaron que no existen efectos tóxicos entre las dosis aplicadas de FC. Los índices de germinación (IG) variaron entre 124,82 a 156,03 \% y los mejores tratamientos fueron para T3 y T2.

Palabras clave: Fibra de coco, agua disponible para las plantas, eficiencia en el uso del agua, alfisol.

\section{INTRODUCCIÓN}

El acelerado incremento de la población mundial y sus proyecciones al alza para el año 2050, significaría alcanzar los 9,6 billones de habitantes en el plane- ta (ONU, 2007), lo que hace necesario la búsqueda de alternativas altamente productivas para abastecer de alimento a la creciente población (Galay, 2009). Es así, como la tecnología de los alimentos ha tenido que ir de la mano con tal incremento (Ramos, 2005), sin embar- 
go, la necesidad de aumentar la producción de alimentos de origen vegetal se ve afectada por la restricción de suelos aptos para la producción agrícola. La escasez y mala calidad del agua, han estimulado a diversos investigadores a buscar alternativas para un desarrollo más rápido y productivo de las distintas especies vegetales (Jeldres, 2000; Lara, 1999).

La eficiencia del uso del agua (EUA) es un aspecto fundamental en la agricultura actual ya que el $70 \%$ del agua es utilizada para este fin (Northoff, 2003). Uno de los objetivos centrales del sector agrícola es producir más alimento con menos agua, lo que se puede lograr aumentando la EUA (Boutraa, 2010). Una de la consecuencias de esto es que se proponen cultivos en invernadero e hidroponía a nivel comercial, que son técnicas de cultivo sin suelo para desarrollar plantas en solución nutritiva (Lara, 1999), en donde se utiliza todo tipo de material, individual o en mezcla de origen natural, de síntesis o residual, mineral u orgánico para ser usado como sustrato en estos medios (Pastor, 1999).

Una de las funciones básicas de los sustratos es abastecer de agua y nutrientes, permitir el intercambio gaseoso en la rizósfera y proporcionar soporte y anclaje a la planta (Pastor, 1999). Su composición puede ser de un solo elemento o bien de una mezcla de estos en caso de no aportar lo necesario, tanto a nivel químico como físico, para el cultivo que se está estableciendo (Abad, 1995).

Adicionalmente, el desarrollo de la industria y el auge de los cultivos sin suelo han generado una creciente necesidad de investigación en sustratos, que buscan satisfacer la demanda por plantas más precoces y productivas (Calderón, 2004). Uno de estos sustratos es la fibra de coco (FC), sustrato poco conocido en Chile, sin embargo, ampliamente utilizado en otras partes del mundo (España, México, Australia y otros). Este sustrato proviene de la familia Palmáceae y de la especie Cocos nucifera L., conocida comúnmente como cocotero o palma de coco, que es uno de los árboles mejor reconocidos y más importantes económicamente en los trópicos (Maruchi et al., 2007). El cocotero es originario de Asia, distribuyéndose principalmente en las áreas tropicales, con una infinidad de usos, siendo fuente entre otros de alimento, bebida, aceite, fibra, combustible, madera, ropa y otros productos (Alfonso y Ramírez, 2008).

La FC es un subproducto de la industria textil, la cual se obtiene del proceso industrial del desecho del tejido del mesocarpio o cáscara del fruto del coco. Este proceso permite obtener fibras largas, cortas y polvo. Los principales países productores de polvo de coco son Sri Lanka, India, Filipinas, Indonesia, México, Brasil, Costa Rica y Guyana (Konduru et al., 1999).

Entre las características que destacan la FC se encuentran su alta capacidad de retención de humedad (sobre 65 \%, según Pire y Pereira, 2003). Vargas et al.
(2008), señalan que el sustrato de FC posee características hidrófilicas, lo que permite una significativa reducción de la cantidad de agua requerida en el riego, obteniéndose una importante disminución en los costos de producción del vivero. El sustrato posee más de un 90 $\%$ de espacio poroso, lo que permite una buena aireación y circulación del agua entre sus poros (Quesada y Méndez, 2005). Tiene un buen equilibrio en la relación aire-agua, posibilitando una mejor respiración de parte de las raíces. Su pH fluctúa entre 5,4 y 6,5, lo que permite una fácil asimilación de todos los elementos nutritivos (Hidalgo et al., 2009). Es amigable con el medio ambiente, siendo un producto de origen orgánico vegetal, que puede ser incorporado al suelo una vez finalizado el cultivo (López-Marín et al., 2008). Al ser un producto de origen vegetal permite una incorporación de materia orgánica al suelo, la que actúa como dosificador del agua, liberando poco a poco la cantidad adecuada para el crecimiento del cultivo, mejorando los rendimientos gracias al mejoramiento de la estructura del suelo (Bonilla et al., 2002). Sin embargo, la salinidad $(0,63$ $\mathrm{dS} \mathrm{m}^{-1}$ ) y variabilidad del origen de la FC, son dos de las principales limitaciones de este sustrato, atribuidos al proceso de producción y a su origen, lo cual puede llegar a producir toxicidad si estas sales se encuentran en una elevada cantidad, aumentando la conductividad eléctrica de la solución (Evans et al., 1996; Vargas et al., 2008). Este aspecto es muy importante, pero se puede evitar fácilmente si en el proceso productivo se realiza un correcto lavado de las fibras, eliminando así los excesos de sales.

El suelo posee características dinámicas (Sandoval et al., 2007) que se pueden mejorar con la incorporación del sustrato de FC, especialmente si estos presentan signos de degradación (p.ej. densidad aparente > $1.2 \mathrm{Mg} \mathrm{m}^{-3}$ o volumen de agua aprovechable para las plantas $<10 \%$, según Reynolds et al., 2008). Algunas de las características del suelo que pueden ser mejoradas con la aplicación de FC son: el contenido de materia orgánica, actividad biológica, estabilidad de agregados, capacidad de almacenaje de agua, capacidad de aireación, velocidad de infiltración, fertilidad del suelo, entre otros (Friedman et al., 2001). En ese contexto, el suelo utilizado en este ensayo (Alfisol) se caracteriza por presentar un avanzado estado de degradación y un escaso aporte de materia orgánica como consecuencia de los efectos climáticos, fuertes pendientes y una intensa actividad agrícola desarrollada por muchos años, lo que ha originado una pérdida en las propiedades físicas, químicas y biológicas que inciden en la sustentabilidad de los suelos, distinguiéndose por la pérdida parcial o total del horizonte A (Sandoval-Estrada et al., 2010; Celis et al., 2011).

Esta investigación plantea como hipótesis que la incorporación del sustrato de fibra de coco en un Alfisol tiene efectos favorables sobre la retención de agua 
aprovechable para las plantas de ballica, mejorando la eficiencia de uso del agua y descartando toxicidad. El objetivo general, fue determinar el efecto de la aplicación de fibra de coco sobre el almacenamiento de agua disponible en un Alfisol y la eficiencia en el uso de esta agua (EUA) por plantas de ballica en condiciones de invernadero. Además, se evaluó el efecto de toxicidad que pudieran provocar las diferentes dosis de fibra de coco utilizada.

\section{MATERIALES Y MÉTODOS}

\section{Ubicación del estudio}

Los ensayos e investigación se realizaron en la temporada 2012 - 2013, en los invernaderos de la Facultad de Agronomía de la Universidad de Concepción, Campus Chillán. En tanto los análisis físico-químicos se realizaron en los laboratorios del Departamento de Suelos y Recursos Naturales de la misma Universidad.

\section{Suelo}

El suelo utilizado se obtuvo del secano interior, sector Huape, VIII región del Bio-Bio a aproximadamente $12 \mathrm{~km}$ al oeste de la ciudad de Chillán. Coordenadas GPS: Latitud $73^{\circ} 89^{\prime} 02^{\prime \prime S}$, Longitud 59 $43^{\prime} 742^{\prime \prime} \mathrm{O}$. Según Stolpe (2006), el suelo corresponde a un Alfisol, asociación Cauquenes de la Cordillera de la Costa (Fine, Kaolinitic, Thermic Ultic Palexeralfs), siendo suelos derivados de rocas graníticas. Las muestras fueron tomadas a $20 \mathrm{~cm}$ de profundidad.

\section{Clima}

El clima predominante en el secano interior corresponde a un mediterráneo marino con una precipitación anual variable de 500 a $1000 \mathrm{~mm}$ (Celis et al., 2007), concentrándose un $80 \%$ del total de agua caída en los meses de Marzo a Agosto y una temperatura anual entre 13,3 y $15,6{ }^{\circ} \mathrm{C}$, siendo el mes más cálido el de enero y el más frío el mes de junio con los máximos en el mes de enero que alcanzan valores de 27 a $31,1^{\circ} \mathrm{C}$ y las temperaturas mínimas van de 3,9 a $5,2^{\circ} \mathrm{C}$ en el mes de junio (Maldonado, 2004).

\section{Sustrato}

El sustrato utilizado fue fibra de coco (FC), originaria de Sri Lanka, importado a Chile a través de Galuku, empresa de origen australiana, y distribuida en Chile por Empresas Protekta LTDA. Según Quesada y Méndez (2005) y Vargas et al. (2008), la FC es un sustrato casi inerte en sus propiedades químicas, destacándose su conductividad eléctrica $\left(0,63 \mathrm{dS} \mathrm{m}^{-1}\right)$ y $\mathrm{pH}$ en agua $(5,63)$. En el aspecto físico se caracteriza por presentar un alto espacio poroso (>90\%), elevada capacidad de retención de humedad (> $65 \%$ ), alto contenido de materia orgánica (> $93 \%$ ) y buen drenaje (Cuadro 1). El sustrato en estudio fue secado a temperatura ambiente y luego tamizado a $2 \mathrm{~mm}$.

\section{Material vegetal}

Las especies utilizadas fueron: (1) Ballica italiana (Lolium multiflorum L.), la que se utilizó para la evaluación de producción de biomasa, por ser una especie de crecimiento vegetativo muy rápido y (2) lechuga (Lactuca sativa L.), la que se usó en pruebas de toxicidad aguda (Sobrero y Ronco, 2004).

\section{Caracterización físico-química del sustrato y del suelo}

La caracterización química de los suelos se realizó mediante las muestras compuestas generadas de las repeticiones para cada tratamiento, mediante la metodología de Sadzawka et al. (2007), mientras que la caracterización física se realizó con metodologías estandarizadas como la curva de retención de humedad, humedad aprovechable, densidad aparente y humedad base peso seco (Sandoval et al., 2012).

\section{Ensayo de biomasa en macetas}

Se realizó en condiciones semi-controladas en invernadero. El ensayo consistió en 12 tratamientos, los cuales consideraron distintas mezclas de fibra de coco y suelo ((FC)/ Suelo) en proporciones de 1:20, 1:40, 1:80 (Cuadro 2). Cada tratamiento consta de tres réplicas, obteniendo 36 unidades experimentales. Además, se incluyeron tres condiciones de humedad distintas (contenido de agua $=100,75$ y $50 \%$ de la capacidad de campo). La reposición del agua se realizó cada 2 días y para el monitoreo del agua a reponer se utilizó un sensor de humedad (Wisebox, N1500FT, Novus, Porto Alegre, Brasil).

Se mezclaron 500 g de suelo (peso seco) por maceta, más la proporción correspondiente del sustrato, previamente tamizados a $2 \mathrm{~mm}$. Luego se sembró 1,0 g de semillas de ballica italiana (Lolium multiflorum L.) 'Nui' con una fertilización en base a urea, superfosfato triple, muriato de potasio y azufre $(0,09 ; 0,053 ; 0,13 \mathrm{y}$ 0,016 g maceta $^{-1}$ ) el 18 de diciembre de 2012. El ensayo se mantuvo durante 3 meses. Cuando las plantas alcanzaron aproximadamente $\operatorname{los} 25 \mathrm{~cm}$ de altura se realizaron los cortes a $2 \mathrm{~cm}$ del suelo (en el período se realizaron un total de 3 cortes y una nueva fertilización base luego de cada corte). La biomasa aérea y radical cosechada se pesó sobre la base peso húmedo y se secó por 48 horas para obtener el peso seco en gramos (g) por maceta en un horno de secado (Memmert, UFB- 
Cuadro 1. Caracterización física y química de la FC y del suelo.

Table 1. Physico-chemical characterization of CF and soil.

\begin{tabular}{|c|c|c|c|}
\hline Análisis Físico - Químico & Unidades & FC & Suelo \\
\hline Porosidad total & $\%$ & 95,00 & 45,00 \\
\hline Densidad aparente (Da) & $\mathrm{g} \mathrm{cm}^{-3}$ & 0,07 & 1,40 \\
\hline Capacidad de campo (CC) & $1 / 3 \mathrm{~atm}(\%)$ & 267,72 & 17,71 \\
\hline Punto marchitez permanente (PMP) & 15 atm (\%) & 28,97 & 9,21 \\
\hline Humedad aprovechable (HA) & $\%$ & 238,75 & 8,50 \\
\hline pH (en agua) & & 5,63 & 5,80 \\
\hline Materia orgánica (MO) & $\%$ & 93,89 & 2,50 \\
\hline Nitrógeno (N) & $\%$ & 16,50 & - \\
\hline Amonio $\left(\mathrm{N}-\mathrm{NH}_{4}\right)$ & $\mathrm{mg} \mathrm{kg}^{-1}$ & 13,80 & 3,30 \\
\hline N-Disponible & $\mathrm{mg} \mathrm{kg}^{-1}$ & - & 9,80 \\
\hline Fósforo (P) & $\%$ & 0,14 & - \\
\hline P-Olsen & $\mathrm{mg} / \mathrm{kg}$ & - & 5,40 \\
\hline Potasio (K) & $\%$ & 1,45 & - \\
\hline Potasio $(\mathrm{K})$ & $\mathrm{mg} \mathrm{kg}^{-1}$ & - & 0,60 \\
\hline Calcio (Ca) & $\%$ & 0,29 & - \\
\hline Calcio (Ca) & $\mathrm{mg} \mathrm{kg}^{-1}$ & - & 5,60 \\
\hline Conductividad eléctrica & $\left(\mathrm{ds} \mathrm{m}^{-1}\right)$ & 0,63 & 0,01 \\
\hline N-Total & $\%$ & 16,50 & 0,15 \\
\hline Relación C/N & & $1 / 100$ & $1 / 9$ \\
\hline
\end{tabular}

Cuadro 2. Tratamientos.

Table 2. Treatments.

\begin{tabular}{|c|c|c|}
\hline Tratamiento & Mezcla & Capacidad de Campo \\
\hline${ }^{1} \mathrm{~T} 0-100$ & $500 \mathrm{~g}$ de suelo & $100 \%$ CC \\
\hline $\mathrm{T} 0-75$ & $500 \mathrm{~g}$ de suelo & $75 \%$ CC \\
\hline $\mathrm{T} 0-50$ & 500 g de suelo & $50 \%$ CC \\
\hline${ }^{2} \mathrm{~T} 1-100$ & $500 \mathrm{~g}$ de suelo $+6,25 \mathrm{~g}$ de FC & $100 \%$ CC \\
\hline $\mathrm{T} 1-75$ & $500 \mathrm{~g}$ de suelo $+6,25 \mathrm{~g}$ de FC & $75 \%$ CC \\
\hline $\mathrm{T} 1-50$ & $500 \mathrm{~g}$ de suelo $+6,25 \mathrm{~g}$ de FC & $50 \%$ CC \\
\hline${ }^{3} \mathrm{~T} 2-100$ & $500 \mathrm{~g}$ de suelo $+12,5 \mathrm{~g}$ de FC & $100 \%$ CC \\
\hline $\mathrm{T} 2-75$ & $500 \mathrm{~g}$ de suelo $+12,5 \mathrm{~g}$ de FC & $75 \%$ CC \\
\hline $\mathrm{T} 2-50$ & $500 \mathrm{~g}$ de suelo $+12,5 \mathrm{~g}$ de FC & $50 \%$ CC \\
\hline${ }^{4} \mathrm{~T} 3-100$ & $500 \mathrm{~g}$ de suelo $+25 \mathrm{~g}$ de FC & $100 \%$ CC \\
\hline T3-75 & $500 \mathrm{~g}$ de suelo $+25 \mathrm{~g}$ de FC & $75 \%$ CC \\
\hline T3-50 & $500 \mathrm{~g}$ de suelo $+25 \mathrm{~g}$ de FC & $50 \%$ CC \\
\hline
\end{tabular}

${ }^{1} \mathrm{~T} 0$ (100-75-50): Suelo solo, 100-75-50 \% de CC; ${ }^{2} \mathrm{~T} 1$ (100-75-50): $1 \mathrm{~g}$ de FC $80 \mathrm{~g}^{-1}$ de suelo y 100-75-50 \% de CC; ${ }^{3} \mathrm{~T} 2$ (100-75-50): $1 \mathrm{~g} \mathrm{de} \mathrm{FC}$ $40 \mathrm{~g}^{-1}$ de suelo y 100-75-50 \% de CC; ${ }^{4} \mathrm{~T} 3$ (100-75-50): 1 g de FC $20 \mathrm{~g}^{-1}$ de suelo y $100-75-50 \%$ de CC. 
500, Medellín, Colombia). La MS total del ensayo se obtuvo de la sumatoria de los tres cortes realizados en el período de estudio.

\section{Determinación de parámetros físico-hídricos de la mezcla}

\section{Gasto de agua}

Corresponde a la sumatoria del agua aplicada durante todo el periodo de ensayo para cada una de las unidades experimentales. De esta forma se determinó el gasto total de agua de cada uno de los tratamientos utilizados.

\section{Eficiencia del uso del agua (EUA)}

Es una forma sencilla de equivalencia de la biomasa o grano, obtenida según el agua que se utilizó durante el desarrollo de la planta. La EUA corresponde a la materia seca total obtenida al final del ensayo dividida por la cantidad de agua aplicada a las macetas (Boutraa, 2010). En general EUA es definida como la relación entre rendimiento del cultivo y el agua aplicada para producir el rendimiento (Howell, 2001; Quezada, 2012):

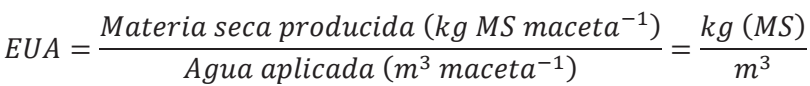

\section{Agua disponible o humedad aprovechable}

Previo al montaje de los ensayos se realizaron curvas de retención de humedad a 1/3 y 15 atmósferas, para la determinación de la capacidad de campo y el punto de marchitez permanente. A partir de esa información se determinó la humedad aprovechable (HA) o máxima capacidad de retención de agua disponible en las distintas mezclas ((FC)/ Suelo) Sandoval et al. (2012).

\section{Preparación de las mezclas suelo / fibra de coco para probar toxicidad}

Se prepararon mezclas de $500 \mathrm{~g}$ de suelo (base seca) con las siguientes proporciones de sustrato: 1:20, 1:40, 1:80 g FC g suelo ${ }^{-1}$ más un control (suelo sin $\mathrm{FC}$ ), equivalentes a 6,0; 3,0 y 1,5 t FC ha-1. Se colocaron en bolsas de polietileno, agregando agua hasta alcanzar el $100 \%$ de capacidad de campo (45, 30 y $20 \mathrm{ml}$ respectivamente/ tratamiento). Las mezclas y el control se incubaron por 15 días en cámara de crecimiento a $25^{\circ} \mathrm{C} \pm 2{ }^{\circ} \mathrm{C}$.

\section{Ensayo de toxicidad aguda en semillas de lechuga}

Con las muestras incubadas por 15 días en las proporciones antes indicadas, se preparó un una mezcla 1:10 (10 g (FC/suelo) y $100 \mathrm{~mL}^{-1}$ de agua destilada). Las mezclas se agitaron a $80 \mathrm{rpm}$ por $30 \mathrm{~min}$. en un agitador recíproco "Heidoph" modelo promax 2020 para su homogenización. Terminado este procedimiento se procedió a colocar $5 \mathrm{~mL}$ de la mezcla en un disco de papel filtro Whatman № 3 ubicado dentro de una cápsula Petri, luego con una pinza se colocaron 20 semillas de lechuga en triplicado por cada uno de los 3 tratamientos, más el testigo (suelo solo) y control (agua destilada).

Enseguida, las cápsulas se llevaron a la cámara de germinación “Trilab”, modelo 01310DZC201, donde se incubaron durante 120 horas a una temperatura de 22 $\pm 2{ }^{\circ} \mathrm{C}$ (Sobrero y Ronco, 2004). A través de las pruebas de germinación se evaluó el índice de germinación (IG), el porcentaje de germinación, la longitud del hipocótilo y la longitud de la radícula de cada una de las plántulas. Esta metodología descrita por Tiquia y Tam (1998), tiene la ventaja de evaluar la toxicidad aguda (que afecta la germinación), así como la toxicidad leve (que afecta el crecimiento de la radícula). El IG se determina a través de la expresión:

$$
I G=\frac{G \times L}{G c \times L c} \times 100
$$

Donde: $\mathrm{IG}=$ índice de germinación (\%); G= promedio de semillas germinadas en la muestra; $\mathrm{Gc}=$ promedio de semillas germinadas en el testigo negativo; $\mathrm{L}=$ promedio de longitud de la radícula en la muestra $(\mathrm{mm})$; Lc= promedio de longitud de la radícula en el testigo negativo ( $\mathrm{mm}$ ).

Se registró el número de semillas que germinaron normalmente, considerando como criterio de germinación la aparición visible de la radícula (5 mm).

\section{Diseño experimental}

El diseño fue completamente al azar, con un arreglo factorial de $4 \times 3$ (tratamiento mezcla suelo-sustrato $\mathrm{x}$ contenido de humedad), resultando 12 tratamientos (incluido un testigo) y tres repeticiones cada uno, por lo que se utilizaron 36 unidades experimentales (Cuadro 2).

\section{Análisis estadístico}

Los datos obtenidos en la investigación fueron analizados para comprobar el supuesto de normalidad con el test de Shapiro-Wilks. Luego, fueron analizados mediante un análisis de varianza (ANDEVA). Al determinar la diferencia entre las medias, se utilizó el test de diferencias mínimas significativas (DMS), P $\leq 0,05$. Para todos los análisis estadísticos se utilizó el programa estadístico computacional SAS (SAS. Institute Inc., 1999).

\section{RESULTADOS Y DISCUSIÓN}

La caracterización del suelo y de la fibra de coco, indican diferencias en cuanto a CC, PMP, HA, Da y MO (Cuadro 1). 
La CC de la FC supera al suelo en 15,1 veces, cifra que resulta superior a otros sustratos (cáscara de arroz $(3,8)$, arena fina $(7,5)$ y bagazo de caña $(9,7)$, según Pire y Pereira, 2003). Estos resultados se ven reflejados en el agua disponible o humedad aprovechable (HA), la cual resulta tener una capacidad 28,1 veces mayor que el suelo de este ensayo. La Da que presenta la FC resultó 20 veces menor que la del suelo, lo que aporta varias ventajas. Según Calderón (2005), un sustrato con baja densidad aparente resulta económicamente beneficioso, debido a que mejora significativamente la capacidad operacional del medio de cultivo, disminuyendo los costos de transporte y manipulación de materiales. La cantidad de MO de la FC supera en 37,55 veces a la del suelo. Este aspecto es fundamental pues la MO actúa como dosificador del agua permitiendo que esta permanezca por más tiempo disponible para las plantas. Además, la alta relación $\mathrm{C} / \mathrm{N}$ que presenta la $\mathrm{FC}$, permite que este producto se mineralice lentamente, resultando una ventaja con relación al almacenamiento de agua en el suelo en el largo plazo. El aporte nutricional de la FC se puede considerar bajo en relación a los niveles normales en análisis de suelos (Vidal, 2007).

\section{Gasto de agua}

Los resultados señalados (Figura 1) muestran diferencias significativas $(P \leq 0,05)$ entre los tratamientos con relación al agua utilizada durante el periodo del ensayo (gasto de agua) para mantener las plantas de ballicas. Los tratamientos sin adición de sustrato (T0) demandaron una mayor cantidad de agua $(\mathrm{P} \leq 0,05)$ durante el periodo al ser comparados con los tratamientos con adición de FC a igualdad de agua a repo- ner, para alcanzar los niveles de CC correspondiente a cada tratamiento. A medida que la mezcla aumenta su contenido de sustrato (FC), esta permite que la humedad permanezca por más tiempo en el suelo a disposición de las plantas. Esto se debe al alto contenido de MO de la FC, añadiendo un mayor espacio poroso al sistema, el cual actúa como dosificador del agua, liberando lentamente la cantidad adecuada de agua para el crecimiento del cultivo (Bonilla et al., 2002). El tratamiento de menor gasto de agua en todo el periodo del ensayo fue el T3-50 (377,8 $\left.\mathrm{mL}^{\text {maceta }}{ }^{-1}\right)$, equivalente al $49 \%$ del agua del tratamiento con mayor gasto (T0100), con una aplicación promedio de $766 \mathrm{~mL} \mathrm{maceta}^{-1}$.

El agua disponible (HA) aumentó cuando se incorporó una mayor cantidad de sustrato a los distintos tratamientos (Cuadro 3), de hecho la FC presentó la mayor HA, resultando el tratamiento sin adición de sustrato el de menor capacidad de almacenamiento. Como se señaló anteriormente, la adición del sustrato al suelo induce un incremento en la porosidad que permite que los tratamientos incrementen su capacidad de retención y contenido de humedad (Pérez et al., 2004).

\section{Eficiencia del uso del agua (EUA)}

La incorporación del sustrato de FC permite un ahorro considerable en el uso del agua, aumentando la eficiencia en el uso de esta (Cuadro 4).

Los tratamientos T3-100, T3-50 y T2-50 alcanzaron una producción de materia seca significativamente mayor ( $\mathrm{P} \leq 0,05$, Cuadro 4 ) y un menor gasto de agua que los demás tratamientos; con excepción de T3-75 que solo fue superado por T3-100, en general los tratamientos controles sin FC, mostraron las menores EUA.

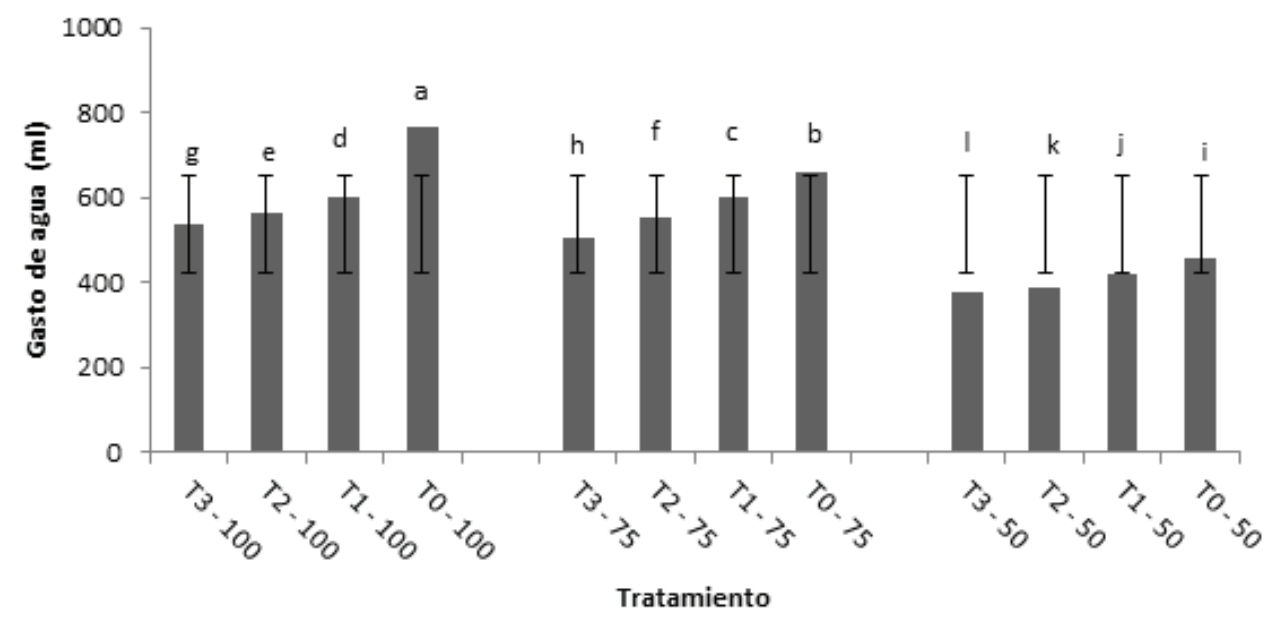

Figura 1. Efecto de la mezcla FC-suelo en el gasto de agua (mL maceta $\left.{ }^{-1}\right)$ en ensayos con siembra de ballica. Figure 1. Effect of CF-soil mixture on water consumption $\left(\mathrm{mL} \mathrm{pot}^{-1}\right)$ in trials with ryegrass seeding.

T0 (100-75-50): Suelo solo, 100-75-50 \% de CC; T1 (100-75-50): $1 \mathrm{~g}$ de fibra de coco $80 \mathrm{~g}^{-1}$ de suelo y 100-75-50 \% de CC; T2 (100-75-50): $1 \mathrm{~g}$ de fibra de coco $40 \mathrm{~g} \mathrm{~g}^{-1}$ de suelo y 100-75-50 \% de CC; T3 (100-75-50): $1 \mathrm{~g}$ de fibra de coco $20 \mathrm{~g}^{-1}$ de suelo y 100-75-50 \% de CC. Letras distintas indican diferencias significativas $(\mathrm{P} \leq 0,05)$. 
Cuadro 3. Humedad aprovechable (HA) para las distintas mezclas FC-suelo.

Table 3. Available moisture (HA) for different CF-soil mixtures.

\begin{tabular}{cccr}
\hline Tratamientos & \% hbss CC (1/3 atm) & $\mathbf{H}^{\mathbf{1}}$ & \%hbss PMP (15 Atm) $^{\mathbf{2}}$ \\
\hline T0 & 17,71 & 9,21 & 8,50 \\
T1 & 20,99 & 10,92 & 10,08 \\
T2 & 23,19 & 12,48 & 10,71 \\
T3 & 28,03 & 14,58 & 13,45 \\
FC & 267,72 & 28,97 & 238,75 \\
\hline
\end{tabular}

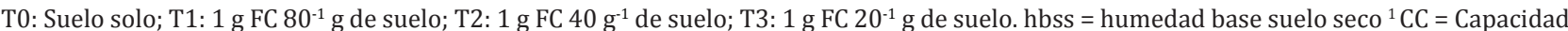
de campo $(1 / 3 \mathrm{~atm}) ;{ }^{2} \mathrm{PMP}=$ Punto de marchitez permanente $(15 \mathrm{~atm}) ;{ }^{3} \mathrm{HA}=\mathrm{CC}-\mathrm{PMP}$.

Cuadro 4. Eficiencia del uso del agua (EUA) para las diferentes mezclas FC-suelo con siembra de ballica italiana.

Table 4. Water use efficiency (EUA) for different mixtures of CF-soil, sowed with Italian ryegrass.

\begin{tabular}{cc}
\hline Tratamiento & EUA (kg MS $\mathbf{~ m}^{-3}$ agua) \\
\hline T3-100 & $8,53 \mathrm{a}$ \\
T3-50 & $8,05 \mathrm{ab}$ \\
T2-50 & $7,99 \mathrm{ab}$ \\
T3-75 & $7,31 \mathrm{bc}$ \\
T2-100 & $6,95 \mathrm{~cd}$ \\
T1-50 & $6,65 \mathrm{cde}$ \\
T2-75 & $6,26 \mathrm{def}$ \\
T1-100 & $5,85 \mathrm{ef}$ \\
T0-50 & $5,47 \mathrm{f}$ \\
T1-75 & $5,27 \mathrm{fg}$ \\
T0-75 & $4,28 \mathrm{hg}$ \\
T0-100 & $4,22 \mathrm{~h}$ \\
C.V & 9,29 \\
DMS & 1,01 \\
\hline
\end{tabular}

T0 (100-75-50): Suelo solo, 100-75-50 \% de CC; T1 (100-75-50): $1 \mathrm{~g}$ de FC $80 \mathrm{~g}^{-1}$ de suelo y 100-75-50 \% de CC; T2 (100-75-50): $1 \mathrm{~g}$ de FC $40 \mathrm{~g}^{-1}$ de suelo y 100-75-50 \% de CC; T3 (100-75-50): $1 \mathrm{~g}$ de FC 20 $\mathrm{g}^{-1}$ de suelo y 100-75-50 \% de CC. C.V: Coeficiente de variación; DMS: Diferencia mínima significativa. Letras distintas indican diferencias significativas $(\mathrm{P} \leq 0,05)$.

Estos antecedentes, muestran la ventaja de incorporar FC para hacer un mejor uso del recurso agua. Es así como los tratamientos con mayor adición del sustrato aumentan la producción de MS $\mathrm{m}^{-3}$ de agua. Northoff (2003), plantea que si en una finca, en países en vías de desarrollo de la zona subsahariana, se mejora el uso efectivo del agua en solo un $1 \%$ se ahorrarán 200 mil litros de agua/ha/día, lo cual se lograría al utilizar el agua en forma correcta, óptima y eficiente. Considerar este aspecto, que es fundamental en la producción agrícola, irá en directo beneficio de los productores, pues el ahorro económico será considerable (Ruiz, 2007). De esta forma, si se logran mejorar las barreras físicas del suelo, como por ejemplo estructuras compactas y alta densidad aparente (Richards et al., 2002) influirá de forma positiva en la EUA.

\section{Ensayo de biomasa en macetas}

\section{Biomasa aérea}

La mejor respuesta a la producción de biomasa aérea con la aplicación de FC al suelo fue el tratamiento T3100 , el cual resultó significativamente mayor $(\mathrm{P} \leq 0,05$, Figura 2) a los demás, con un $45 \%$ más de biomasa (expresada como materia seca) al compararlo con el tratamiento que menos biomasa produjo (T0-50). La mayor producción de biomasa se debe principalmente al aporte de FC, la cual genera mejoras en las condiciones físicas del suelo que sumado a sus propiedades específicas, como son el alto contenido de materia orgánica, baja densidad aparente, alta porosidad y a la alta capacidad de retención y acumulación de humedad (Di Benedetto et al., 2000), permite a las plantas mejores respuestas fisiológicas y productivas, ya que las raíces tienen un espacio adecuado para la obtención de nutrientes y un mejor intercambio gaseoso y radical (Calderón, 2004).

En la Figura 2 se verifica que con un incremento en la dosis de FC y contenidos de agua cercanos a capacidad de campo se obtuvo una mayor producción de biomasa áerea (T3-100, T2-100, T3-75 y T1-100). Éstos resultados son coincidentes con un estudio en plantas ornamentales realizado por Meerow (1994), quien reportó diferencias significativas al comparar distintos sustratos (turba y FC) en donde el uso de la FC mostró mayores crecimientos en las plantas. 


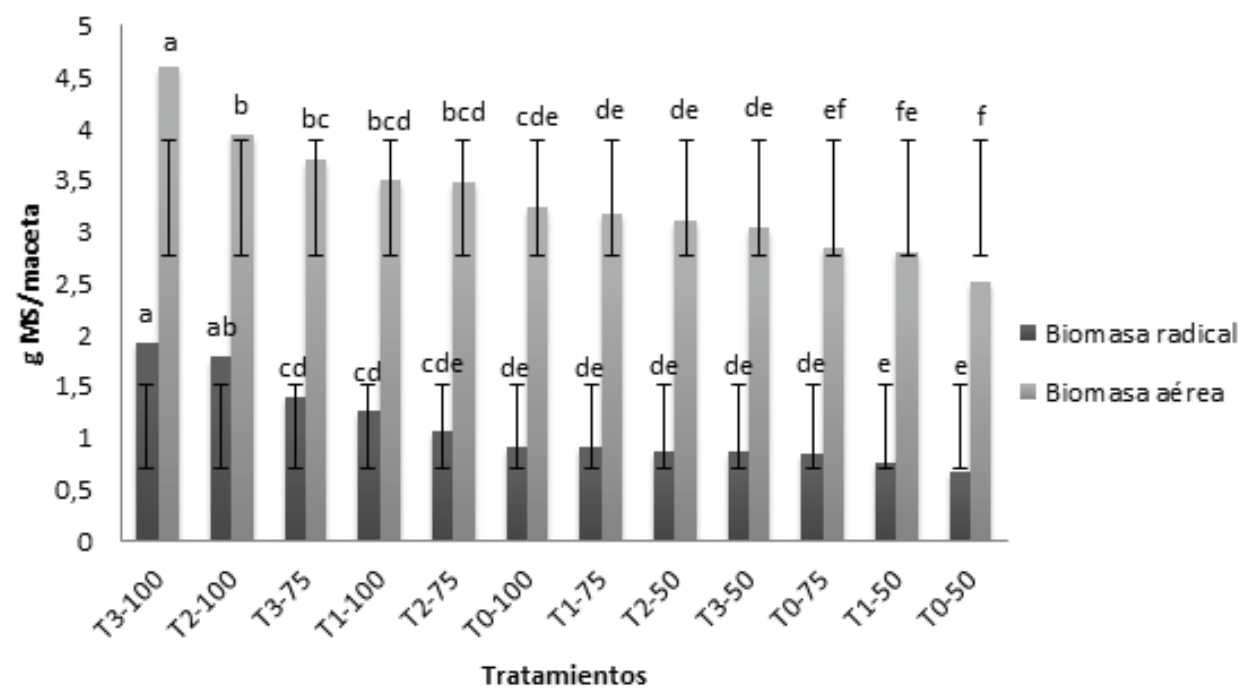

Figura 2. Biomasa aérea y radical de ballica italiana como respuesta a las diferentes mezclas de FC-suelo y diferentes reposiciones de agua en relación al contenido de agua del suelo a 1/3 atmosfera (capacidad de campo).

Figure 2. Aereal and root biomass of Italian ryegrass in response to the different mix of CF-soil and different water replenishment in relation to soil water content at $1 / 3$ atmosphere (field capacity).

T0 (100-75-50): Suelo solo, 100-75-50 \% de CC; T1 (100-75-50): $1 \mathrm{~g}$ de fibra de coco $80 \mathrm{~g}^{-1}$ de suelo y 100-75-50 \% de CC; T2 (100-75-50): $1 \mathrm{~g}$ de fibra de coco $40 \mathrm{~g}^{-1}$ de suelo y 100-75-50 \% de CC; T3 (100-75-50): $1 \mathrm{~g}$ de fibra de coco $20 \mathrm{~g}^{-1}$ de suelo y 100-75-50 \% de CC. Letras distintas indican diferencias significativas $(\mathrm{P} \leq 0,05)$.

\section{Biomasa radical}

La Figura 2 también muestra tendencias similares en cuanto a la acumulación de biomasa aérea. Se destaca que los tratamientos con la mayor cantidad de sustrato e independientemente de la cantidad de agua aplicada (T3-100 y T2-100) fueron los que obtuvieron la mayor producción de biomasa radical, resultando el tratamiento $\mathrm{T} 3-100$ significativamente mayor $(\mathrm{P} \leq 0,05)$ que los demás tratamientos e igual que T2-100.

El tratamiento testigo (T0-50), resultó con un 65 $\%$ menos de producción de biomasa radical al compararlo con el tratamiento más efectivo (T3-100). Esto, es atribuible a los mismos factores mencionados anteriormente (MO, Da, HA, porosidad), lo que significa que las plantas en estos tratamientos no tuvieron un estrés hídrico importante durante el período que duró el ensayo, pues siempre se mantuvieron con la humedad adecuada, lo que permitió a las plantas soportar de mejor manera las condiciones ambientales dadas en condiciones de invernadero, mejorando el entorno de las raíces y el crecimiento de las plantas. Es así como Ellies (2004), señaló que cuando se mejora y regula el entorno de las raíces estas obtendrán mayores beneficios en cuanto a nutrientes y capacidad de aireación dentro de su entorno. Así, un espacio poroso adecuado para las raíces vendrá en directo beneficio para la obtención de nutrientes y para una adecuada respiración radical (Calderón, 2005).

\section{Ensayo de toxicidad aguda en semillas de lechuga}

Los valores de IG obtenidos con la aplicación de las mezclas de suelo con FC variaron de 124,82 a 156,03 $\%$ (Cuadro 5).

Según Schleef et al. (2004), un IG sobre $60 \%$ no presenta un efecto fitotóxico en la germinación de semillas. De este modo, los resultados obtenidos con T1, T2 y T3 pueden ser considerados no fitotóxicos para todos los tratamientos, los cuales a la vez pueden llegar a ser considerados hasta estimulantes para la germinación y elongación tanto de radículas como de los hipocótilos de las plántulas. Otros estudios con distintos sustratos, como residuos de lodos urbanos y de salmonicultura descritos por Celis et al. $(2007 ; 2008)$ no presentaron fitotoxidad con aplicaciones de $90 \mathrm{t} \mathrm{ha}^{-1}$, entendiendo que estos residuos por su origen son de composición y riesgo mucho mayor a residuos de origen vegetal como es la FC.

Entre los tratamientos con FC no se observaron diferencias significativas $(P \leq 0,05)$ para el IG, pero en el caso de los tratamientos T2 y T3 si existieron diferencias significativas $(P \leq 0,05)$ con el tratamiento testigo (T0). El tratamiento T1 no muestra diferencias significativas con T0, esto debido a su bajo contenido de FC en las mezclas. Este menor contenido del sustrato (FC) en T1 no alcanza a generar diferencias significativas con el testigo, pero si se originan diferencias con los tratamientos con mayor aporte de sustrato, siendo estos últimos los que alcanza el mayor IG en los tratamientos. 
Cuadro 5. Efecto de la mezcla FC - suelo sobre el Índice de Germinación (IG) y longitud radicular (mm planta-1) en lechuga.

Table 5. Effects of CF-soil mixture on Germination Index (GI) and root length ( $\mathrm{mm} \mathrm{plant}^{-1}$ ) in lettuce.

\begin{tabular}{lcc}
\hline Tratamientos & Índice de Germinación (\%) & Longitud radicular (mm) \\
\hline T3 & $156,03 \mathrm{a}$ & $25,70 \mathrm{a}$ \\
T2 & $144,77 \mathrm{a}$ & $25,70 \mathrm{a}$ \\
T1 & $124,82 \mathrm{ab}$ & $21,77 \mathrm{~b}$ \\
T0 & $100,00 \mathrm{~b}$ & $18,15 \mathrm{c}$ \\
C.V. & 12,87 & 6,63 \\
D.M.S & 33,78 & 3,02 \\
\hline
\end{tabular}

T0: Suelo solo; T1: 1 g de FC $80 \mathrm{~g}^{-1}$ de suelo; T2: 1 g de FC $40 \mathrm{~g}^{-1}$ de suelo); T3: 1 g de FC $20 \mathrm{~g}^{-1}$ de suelo); C.V: Coeficiente de variación; D.M.S: Diferencia mínima significativa. Letras distintas indican diferencias significativas $(\mathrm{P} \leq 0,05)$.

La longitud radicular de las semillas de lechuga en los tres tratamientos con FC fue superior que T0 o control (Cuadro 5), observándose diferencias significativas con este $(\mathrm{P} \leq 0,05)$ sin presentar ningún tipo de efecto tóxico sobre la elongación de la raíz. Además se aprecia que no existen diferencias significativas $(P \leq 0,05)$ entre los tratamientos $\mathrm{T} 3$ y $\mathrm{T} 2$, pero si con los tratamientos T1 y T0. En consecuencia, las pruebas de fitotoxicidad aplicadas demuestran que la FC, no generó efectos negativos con las dosis utilizadas en este ensayo.

\section{CONCLUSIONES}

Todos los tratamientos que incluyeron fibra de coco (FC) generaron un ahorro significativo en la cantidad de agua a reponer, de hecho el mejor uso eficiente del agua lo obtuvieron los tratamiento con las mayores dosis de FC incorporada al suelo, y asociados a mantener un 100 y $50 \%$ de la capacidad de campo (T3-100, T350 y T2-50). En consideración a la sustentabilidad del recurso agua, deberían preferirse aquellos tratamientos que mantuvieron un $50 \%$ de la CC (T3-50, T2-50). En estos tratamientos, también se vio incrementada la biomasa aérea y radical. El aumento de la FC, mejoró la capacidad de almacenamiento de agua y disminuyó la densidad aparente. En relación a la toxicidad de la FC esta fue descartada.

\section{REFERENCIAS}

ABAD, M. 1995. Sustratos para cultivos sin suelo. Nuez, F. (Ed.). En: El cultivo del tomate. Ediciones Mundi-Prensa. Madrid, España. pp: 131-166.

ALFONSO, J., RAMÍREZ, T. 2008. Manual técnico del cultivo del cocotero (Cocos nucífera L.). Cuenta del Desafío del Milenio, Honduras. Disponible en: http://www.mcahonduras.hn/documentos/publicacioneseda/Manuales\%20de\%20produccion/EDA_Manual_Produccion_ Coco_FHIA_09_08.pdf. [Consulta: 10 junio 2013].
BONILLA, C., BONOMELLI, C., URRUTIA, G. 2002. Distribución espacial y temporal de la precipitación y la humedad del suelo en tres sitios forestales de la VIII Región de Chile. Agricultura Técnica (Chile) 62(4): 541-554.

BOUTRAA, T. 2010. Improvement of water use efficiency in irrigated agriculture: a review. Journal Agronomy 9(1): 1-8.

CALDERÓN, A. 2004. Propiedades físicas de los sustratos Proyecto Fondef. Universidad de Chile, Fac. Cs. Agronómicas. 3 p.

CALDERÓN, A. 2005. Sustratos agrícolas Proyecto Fondef. Universidad de Chile, Fac. Cs. Agronómicas. 4 p.

CELIS, J., SANDOVAL, M., BRIONES, M. 2007. Bioensayos de fitotoxicidad de residuos orgánicos en lechuga y ballica anual realizados en un suelo Alfisol degradado. Revista de la Ciencia del Suelo y Nutrición Vegetal 7(3): 51-60.

CELIS, J., SANDOVAL, M., BARRA, R. 2008. Plant response to salmon wastes and sewage sludge used as organic fertilizer on two degraded soils under greenhouse conditions. Chilean Journal Agricultural Research 68(3): 274283.

CELIS, J., SANDOVAL, M., BELLO, N. 2011. No-linear respiration dynamics in a degraded Alfisol amended with different dose of salmon sludges. Journal Soil Science Plant Nutrition 11(1): 58-67.

DARWISH, T., ATALLAH, T., HAJHASAN, S., HAIDAR, A. 2006. Nitrogen and water use efficiency of fertigated processing potato. Agricultural Water Management 85(1-2): 95-104.

DI BENEDETTO, A., MOLINARI, J., BOSCHI, C., KLASMAN, R., BENEDICTO, D. 2000. Adaptación de cuatro especies florales anuales a diferentes substratos de crecimiento. Agro Sur 28(2): 69-76.

ELLIES, A. 2004. Efecto de la materia orgánica sobre la es- 
tructura del suelo. Boletín $\mathrm{N}^{\circ} 20$. Sociedad Chilena de la Ciencia del Suelo / Universidad de La Frontera. Temuco, Chile. pp: 139-150.

EVANS, M., KONDURU, S., STAMPS, R. 1996. Source variation in physical and chemical properties of coconut coir dust. HortScience 31(6): 965-967.

FRIEDMAN, D., HUBBS, M., TUGEL, A., SEYBOLD, C., SUCIK, M. 2001. Guidelines for soil quality assessment in conservation planning. United States Department of Agriculture. Disponible en:<http://www.nrcs.usda.gov/Internet/FSE_DOCUMENTS/nrcs143_019133.pdf.> [Consulta: 10 agosto 2013].

GALAY, C. 2009. Derecho a la alimentación y acceso a la justicia: ejemplos a nivel nacional, regional e internacional FAO. Disponible en: <http://www.fao.org/docrep/016/ k7286s/k7286s.pdf>. [Consulta: 09 octubre 2012].

HIDALGO, P., SINDONI, M., MARÍN, C. 2009. Evaluación de sustratos a base de vermicompost y enmiendas orgánicas líquidas en la propagación de parchita (Passiflora edulis v. flavicarpa) en vivero. Rev. Cient. UDO Agríc. 9(1): 126135.

HOWELL, T.A. 2001. Enhancing water use efficiency in irrigated agriculture. Agronomy Journal 93(2): 281-289.

JELDRES, D. 2000. Manejo y conservación de suelos y aguas en microcuencas hidrográficas. Boletín INIA № 42. INIA Quilamapu. Chillán. pp: 43-76.

KARLEN, D., MAUSBACH, M., DORAN, J., CLINE, R., HARRIS, R., SCHUMAN, G. 1997. Soil quality: a concept, definition, and framework for evaluation. Soil Science Society of America Journal 61(1): 4-10.

KONDURU, S., EVANS, M., STAMPS, R. 1999. Coconut husk and processing effect on chemical and physical properties of coconut coir dust. HortScience 34(1): 88-90.

LARA, A. 1999. Manejo de la solución nutritiva en la producción de tomate en hidroponía. Terra Latinoamericana 17(3): 221-229.

LÓPEZ-MARÍN, J., GÁLVEZ, A., RODRÍGUEZ, C.M., CONESA, E., OCHOA, J., GONZÁLEZ, A. 2008. Utilización de la fibra de coco como sustrato alternativo a las turbas en cultivo de clavel para maceta Sociedad Española de Agricultura Ecológica. Disponible en:<http://www.agroecologia. net/recursos/congresos/bullas08/SEAE_LLIBRE_resums_BULLAS.pdf/>. [Consulta: 09 noviembre 2012].

MALDONADO, I. 2004. El clima en el área agroecológica del secano interior. Boletín INIA № 123. INIA Quilamapu. Chillán. pp: 1-15.

MARUCHI, A., CUETO, J., SANTOS, Y., ROMERO, W., LLAUGER, R., ROHDE, W. 2007. Variabilidad morfológica y molecular de una población de cocoteros verdes en la Región de
Baracoa. Cultivos Tropicales 28(3): 69-75.

MEEROW, A. 1994. Growth of two subtropical ornamentals using coir (Coconut mesocarp pith) as a peat substitute. HortScience 29(12): 1484-1486.

NORTHOFF, E. 2003. No hay crisis mundial de agua, pero muchos países en vías de desarrollo tendrán que hacer frente a la escasez de recursos hídricos FAO. Disponible en:<http://www.fao.org/spanish/newsroom/ news/2003/15254-es.html>. [Consulta: 17 noviembre 2012].

ONU (USA). 2007. ONU estima que población mundial llegará a 9.000 millones en 2050. ONU. Disponible en: < http://www.un.org/spanish/News/story. asp?newsID=9007\&criteria1=\#.U_Id5fldVAo>. [Consulta: 03 octubre 2012].

PASTOR, J. 1999. Utilización de sustratos en viveros. Terra Latinoamericana 17(3): 231-235.

PÉREZ, A., REGALADO, C., CID, M. 2004. Consecuencias hidrológicas de la compactación en fibra de coco. Instituto Canario de Investigaciones Agrarias, España. Disponible en: <http://www.icia.es/icia/index.php?option=com_ content\&view=article\&id=3040: consecuencias-hidrologicas-de-la-compactacion-en-fibra-de-coco\&catid $=14$ 5\&Itemid=262\&lang=en > [Consulta: 03 julio 2013].

PIRE, R., PEREIRA, A. 2003. Propiedades físicas de componentes de sustratos de uso común en la horticultura del Estado Lara, Venezuela. Propuesta metodológica. Bioagro 15(1): 55-64.

QUESADA, G., MÉNDEZ, C. 2005. Análisis fisicoquímico de materias primas y sustratos de uso potencial en almácigos de hortalizas. Revista Agricultura Tropical 35: 1-13.

QUEZADA, L.C. 2012. Requerimientos de riego. En: Fundamentos de riego. Publicaciones Departamento de Suelos y Recursos Naturales $\mathrm{N}^{\circ} 6$. Universidad de Concepción. Chillán. pp: 35-50.

RAMOS, C. 2005. Residuos orgánicos de origen urbano e industrial que se incorporan al suelo como alternativa económica en la agricultura. Revista CENIC. Ciencias Químicas 36(1): 45-53.

REYNOLDS, W.D., DRURY, C.F., TAN, C.S., FOX, C.A., YANG, X.M. (2009): Use of indicators and pore volume-function characteristics to quantify soil physical quality. Geoderma 152: 252-263.

RICHARDS, R., REBETZKE, G., CONDON, A., VAN HERWAARDEN, A. 2002. Breeding opportunities for increasing the efficiency of water use and crop yield in temperate cereals. Crop Science 42(1): 111-121.

RUIZ, J. 2007. Servicios ambientales, agua y economía. Revista de Ingeniería. (26): 93-100. 
SADZAWKA, R., CARRASCO, M., DEMANET, R., FLORES, H., GREZ, R., MORA, M., NEAMAN, A. 2007. Métodos de análisis de tejidos vegetales (2a. ed.). Serie Actas INIA № 40. INIA La Platina. Santiago.

SANDOVAL, M., CASTILLO, C., ZAGAL, E., STOLPE, N., UNDURRAGA, P. 2007. Parámetros hidráulicos determinados de un Andisol bajo diferentes rotaciones culturales después de diez años. Revista de la Ciencia del Suelo y Nutrición Vegetal 7(2): 32-45.

SANDOVAL, M., DÖRNER, J., SEGUEL, O., CUEVAS J., RIVERA, D. 2012. Retención del agua en el suelo. En: Métodos de análisis físicos de suelos. Publicaciones Departamento de Suelos y Recursos Naturales $N^{\circ} 5$. Universidad de Concepción. Chillán. pp: 65-77.

SANDOVAL-ESTRADA, M., CELISHIDALGO, J., STOLPE-LAU, N., CAPULÍN- GRANDE, J. 2010. Efecto de enmiendas con lodos urbanos y de salmonicultura en la estructura de un Entisol y un Alfisol en Chile. Agrociencia 44(5): 503-513.

SAS INSTITUTE. The SAS® system for Windows [cd-rom]. Ver. 8. Cary: 1999. 1 cd-rom.

SCHLEEF, E., VERGARA, C., DIEZ, M. 2004. Evaluación de alternativas para la estabilización de biosólidos generados en un sistema de tratamiento de aguas servidas. Boletín
№ 20. Sociedad Chilena de la Ciencia del Suelo / Universidad de La Frontera. Temuco. pp: 39-51.

SOBRERO, M., RONCO, A. 2004. Ensayo de toxicidad aguda con semillas de lechuga (Lactuca sativa L.). Centro Internacional de Investigaciones para el Desarrollo, México. Disponible en:<http://www2.inecc.gob.mx/ publicaciones/libros/573/cap4.pdf.> [Consulta: 12 septiembre 2013].

STOLPE, N. 2006. Grupo de suelos: graníticos. En: Descripciones de los principales suelos de la VIII Región de Chile. Publicaciones Departamento de Suelos y Recursos Naturales. Universidad de Concepción. Chillán. pp: 48-52.

TIQUIA, S., TAM, N. 1998. Elimination of phytotoxicity during co-composting of spent pig-manure sawdust litter and pig sludge. Bioresource Technology 65(1-2): 43-49.

VARGAS, P., CASTELLANOS, J., SÁNCHEZ, P., TIJERINA, L., LÓPEZ, R., OJODEAGUA, J. 2008. Caracterización física, química y biológica de sustratos de polvo de coco. Revista fitotecnia mexicana 31(4): 375-381.

VIDAL, I. 2007. Nutrición mineral y diagnóstico del estado nutricional de las plantas. En: Fertirrigación, cultivos y frutales. Publicaciones Departamento de Suelos y Recursos Naturales. Universidad de Concepción. Chillán. pp: 7-21. 
